\begin{tabular}{ll} 
LATE-PCR arrives in time & $\mathbf{8 7 0}$ \\
\hline RNA: the uncertainty principle & $\mathbf{8 7 1}$ \\
\hline A brave new digital world & $\mathbf{8 7 1}$ \\
\hline The heart of personal genomics & $\mathbf{8 7 2}$ \\
\hline Box 1: The numbers game & 870 \\
\hline
\end{tabular}

\title{
PCR's next frontier
}

\section{PCR - the workhorse of modern molecular biology — is charging forward using both conventional and} digital methods to explore single cells and even single molecules. Nathan Blow reports.

In late 1978, Ry Cooder recorded an album entitled Bop till you drop entirely in a new digital format. It was the first popular music album to forego the conventional analog format, and within a couple of years more musicians began to see the value of digital, which has now replaced analog altogether.

Molecular biology's equivalent of Cooder's Bop till you drop just might be digital PCR. This technique's aim is absolute quantitation of molecules in a sample by counting them directly, as opposed to inferring the amount of starting material from an amplified product, which conventional PCR does. The quantitation in digital PCR is achieved by diluting the starting sample so that there is at most only one molecule in each well. After amplification, the number of wells containing the product is counted to determine the number of molecules in the starting sample.

"With digital approaches, you can even simply add up sequence tags from a number of experiments the lab does or another lab does, and you can directly compare results even though they are not part of the same experiment," explains Bert Vogelstein of Johns Hopkins University. But this technology is still in its infancy, "just like 'digital' was recently in music, so there are some problems that need to be worked out," says Vogelstein.

In recent years, both conventional and digital PCR methods have found use in singlecell and single-molecule genetic analysis. As scientists and companies refine conventional real-time PCR methods to perform semiquantitative analysis of RNA transcripts from single cells, digital methods are also plowing forward, providing quantitative transcription profiling and serving as the basis for several next-generation sequencing systems.

So will PCR leave its analog roots and go completely digital? Or will digital be nothing more than a 'fad' in the genetics world?

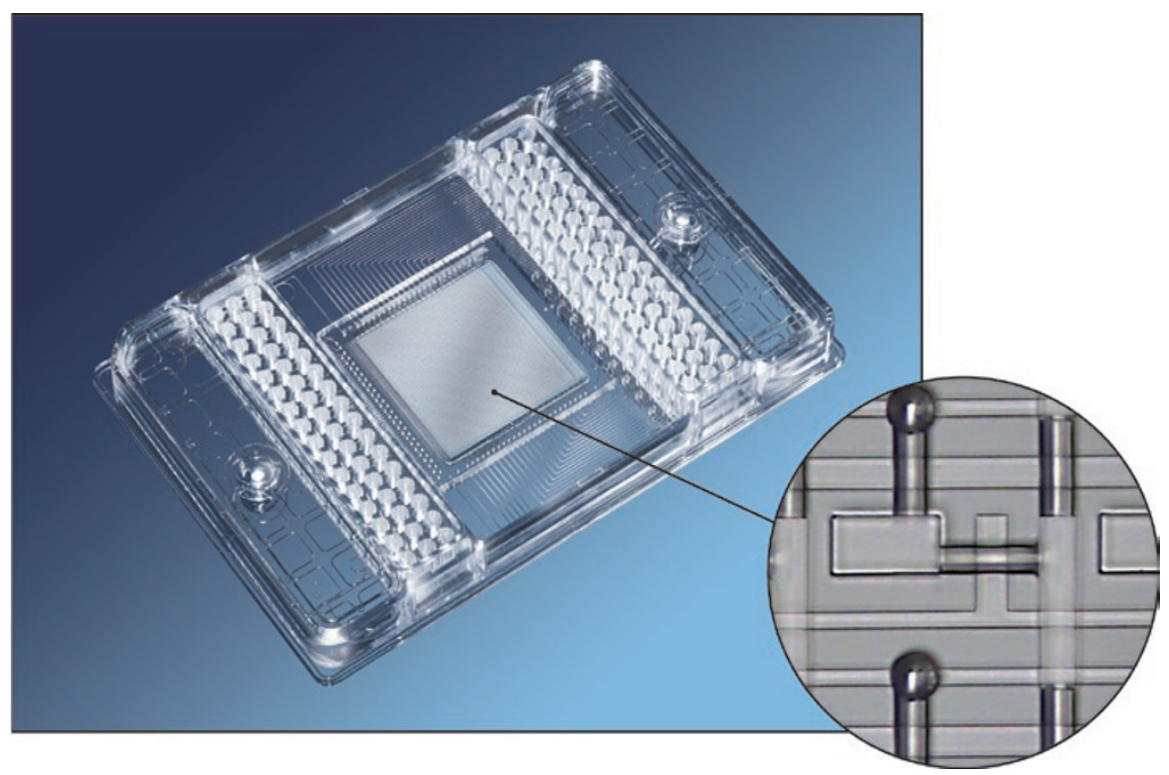

Fluidigm offers several array-based solutions for conventional and digital PCR. (Image courtesy of Fluidigm.)

The answers to these questions just might lie in the experiences of developers advancing conventional and digital PCR.

\section{The single life}

Real-time PCR uses either fluorescencebased probes or dyes to detect amplified products. Fluorescence-based probes—such as molecular beacons, TaqMan probes and Scorpion probes - generally have a fluorescent reporter dye on one side and a quencher on the other. During amplification these probes either decouple or change conformation, emitting fluorescence that can be directly measured.

Dyes such as SYBR Green bind doublestranded DNA and emit light upon excitation. During PCR fluorescence increases as the amplification products accumulate. With SYBR Green there is no probe that has to be synthesized, but the use of a dye lacks the sequence-specificity of probe- based methods as any generated product, or primer-dimer, will fluoresce.

Invitrogen now supplies an improved version of their SYBR Green dye, called SYBR GreenER, which may eliminate some background fluorescence problems associated with the old formulation. Sandrine Javorschi-Miller, program manager of gene regulation at Invitrogen claims that the new enzyme formulation in SYBR GreenER helps reduce the risk of primer-dimer formation at the beginning of the reaction because at room temperature the enzyme has no residual activity, and the new dye causes less PCR inhibition. "People tend to think it is the dye or the enzyme, but really it is a combination of both," says JavorschiMiller when discussing the effectiveness of SYBR GreenER.

One of the most challenging problems real-time PCR has faced in recent years is the difficulty of working with very small 
amounts of template or even DNA from single cells. "If you are looking at a singlecopy gene in a single cell it gets very difficult to detect it nicely," says Javorschi-Miller. Although amplification of DNA from single cells using whole-genome amplification approaches has proven helpful, this has not solved the entire problem as these methods can cause amplification bias (see Box 1).

\section{LATE-PCR arrives in time}

Larry Wangh of Brandeis University got interested in single-cell PCR analysis through his work in a genetic counseling program. "During teaching I realized that pre-implantation genetic diagnosis could be used as an alternative to prenatal diagnosis, which sometimes causes couples quite a complicated decision-making process," says Wangh.
This interest in genetic diagnosis and his work with single cells from human embryos led Wangh to develop linear-after-theexponential (LATE)-PCR ${ }^{1}$, which he describes as an improved form of asymmetric PCR. The problem with asymmetric $\mathrm{PCR}$, which requires dilution of one of the two primers to make it a limiting primer, is that the decrease in the melting temperature of that limiting primer upon dilution makes it less efficient in the reaction. To remedy this situation, the annealing temperature of the reaction had to be decreased, increasing the probability of mispriming, says Wangh. By simply increasing the melting temperature of the limiting primer, Wangh found that this problem could be overcome and specificity increased.

\section{BOX 1 THE NUMBERS GAME}

When starting with a sample as small as a single cell, PCR amplification can prove difficult. "There is a huge difference between single cells and a small number of cells," says Gerald Schock, global product manager of whole genome amplification and epigenetics at Qiagen. "We realized that sufficient DNA is not available from single cells for many applications." Researchers and companies alike have started tackling this problem by developing methods for whole-genome amplification.

There are essentially two amplification approaches: one uses PCR with a polymerase such as Taq and the other is multiple displacement amplification using the Phi29 polymerase. PCR-based methods for amplification, such as Sigma-Aldrich's GenomePlex systems, rely on primers for amplification of the genome using Taq polymerase. The GenomePlex single-cell whole amplification kit works by first fragmenting genomic DNA into short overlapping templates with defined $5^{\prime}$ and $3^{\prime}$ fixed ends that can be used for isothermal amplification and subsequent standard PCR to generate amplified DNA. "When starting with single-cell material and doing a single-cell amplification, we have demonstrated that there is limited amplification bias using the GenomePlex system," says Shannon Dempsey, product manager at Sigma-Aldrich.

Schock also acknowledges DNA polymerases can have some degree of bias. "We had feedback from researchers, that you see a change in the locus representation due to PCR-based whole genome amplification." This is the reason that Qiagen and other companies, such as GE Healthcare, are also exploring linear amplification. "The Phi29 is a multi-displacement enzyme where the bias is very low," says Schock. But he adds that what bias is found when amplifying with Phi29 is strongly influenced by the DNA preparation method and amount of starting material. The key is to avoid breaks in the DNA template by using a gentle denaturation procedure during whole-genome amplification, and Qiagen scientists have worked on defining the optimal conditions for extracting DNA from even single cells to minimize strand breakage or damage. "The more break points and lower DNA amounts, the less likely it is that you will be able to detect your locus of interest," says Schock.

For Fluidigm a simple pre-amplification step has proved very helpful when combined with their real-time PCR dynamic arrays. "Preamplification is a fairly new PCR technique that allows you to amplify just the target genes that you wish to measure quantitatively," says Mike Lucero. Pre-amplification uses a limited dilution of primers and only ten cycles of PCR so that the reaction does not enter the plateau phase, explains Lucero. "With pre-amplification you get a 500-fold increase in the transcripts, but preserve the relationships between the transcripts you wish to measure," says Lucero. 
Wangh and his colleagues have since gone on to demonstrate the usefulness of LATEPCR in amplifying DNA from single cells. During the development of LATE-PCR, they also serentipitously identified a new class of molecules that increase PCR amplification specificity from single cells. "We were making a molecule and put it into the reaction and suddenly the reaction got crisper," recalls Wangh. Called PrimeSafe and now marketed by Smiths Detection, these small molecules improve PCR specificity by inhibiting polymerases in a temperaturedependent manner. At lower temperatures the molecules inhibit polymerase activity, but at higher temperatures the inhibition is completely relieved.

\section{RNA: the uncertainty principle}

RNA amplification at the single-cell level using conventional methods is even more complicated than DNA amplification because amounts of mRNA transcripts vary widely between cells. "Basically it is possible to quantitate levels of mRNA in cells using currently available methods; however, there is a problem of uncertainty," says Sanjay Tyagi from Public Health Research Institute Center. "PCR is an amplification process and when you have very low numbers of RNA you get stochastic fluctuations from having so few molecules," says Tyagi.

Other factors, such as the use of reverse transcriptase to convert mRNA to cDNA, which Tyagi says can be as low as 30\% effective, exacerbate the problem. Companies go have been trying to solve this reverse transcription problem by using enzymes such as SuperscriptIII from Invitrogen, which is a mutant enzyme with reduced the RNase $\mathrm{H}$ activity to make reverse transcription more efficient.

In addition to these fluctuations in the amplification process, there are fluctuations in gene expression, an area that Tyagi's lab has been studying in detail. He recently demonstrated that for some genes, the variation in mRNA production can be quite large 2 ."We found that there was massive variation-most cells produced nothing but some produced a lot of RNA — and that the RNA was being transcribed in bursts," explains Tyagi. The question of the biological importance of the variation and even the amount of variation is wide open. "We don't know the full scope of RNA variation in cells," says Tyagi, "but if the variation was on the level that we have described, things just won't function properly."

\section{A brave new digital world}

Digital PCR, first described in 1997 by Olga Kalinina and colleagues, requires diluting samples of molecules over an entire multiwell plate with the goal of having either a single molecule or no molecules in each well. PCR can then be performed and probes added to each well, detecting if amplification occurred or not—a very simple plus or minus result.
Tyagi believes that digital PCR actually has dual benefits. He points to the fact that at limiting dilutions not only do the target molecules end up in different wells, the unrelated DNA molecules that generate false amplicons are also segregated. In this way it is possible to increase the number of cycles during digital PCR, increasing the likelihood of amplification of a molecule if it is in a well. 
In February 2007 Fluidigm introduced a new array capable of performing digital PCR. "We have a chip that is the embodiment of digital PCR," claims Mike Lucero executive vice president of sales and marketing at Fluidigm. Using the Fluidigm digital array, up to 12 different reactions with template, primers and probes can be partitioned into 765 separate chambers, and PCR can be performed for each. This results in a signal for each well confirming whether amplification occurred in that particular chamber or not. By simply counting the number of 'positive' chambers researchers can determine the starting number of molecules in the sample.

Another benefit of the Fluidigm digital array is that real-time curves can be generated for each chamber if necessary. This allows users to determine whether multiple molecules were partitioned into any particular chamber, thereby eliminating uncertainty if the number of starting molecules was greater than 765-the number of separate chambers.

The generation of real-time curves for large numbers of chambers has its origins in another Fluidigm product, the dynamic array, which is used for conventional real-time PCR. The dynamic array can generate real-time curves for 48 different transcripts from 48 different single cell samples. "Each run produces 2,304 real-time curves," says Mike Lucero, "so it really comes in handy for applications like single-cell analysis where you have a limiting sample.” Lucero is quick to point out that with the digital array researchers are getting the best of both worlds.

\section{BEAM me up!}

Digital PCR methods initially allowed researchers to look at a couple hundred molecules on a microtiter plate. "This was useful but what you really want to be able to do is look at millions of molecules to get statistically significant data," says Vogelstein, who developed a method called BEAMing, "And what BEAMing does is basically provide millions of wells in a single test tube."

BEAMing stands for beads, emulsion, amplification and magnetics. In the assay, a population of DNA fragments is pre-amplified; then individual beads bound with primers are confined with pre-amplified templates into individual compartments of a water-in-oil emulsion. The single molecules are amplified

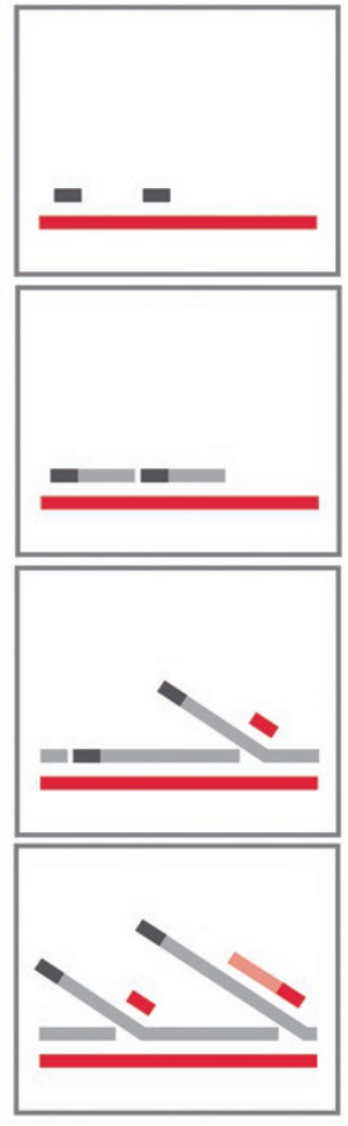

The Qiagen Repli-G system allows wholegenome amplification by first annealing primers to template DNA and then using the Phi29 polymerase for linear amplification. (Image courtesy of Qiagen.)

on the bead by PCR. Finally the emulsion is broken, transforming a population of molecules to a population of beads in a roughly $1: 1$ manner.

According to Vogelstein, BEAMing is a robust technology: anything you can do with a single molecule, any enzymatic or hybridization reaction, you can do with the beads once you have converted them through BEAMing. Vogelstein's group, which is interested in developing diagnostic tests for cancer, has applied the technology to search for rare mutations that are present in the blood of cancer patients ${ }^{3}$.

\section{The heart of personal genomics}

George Church of Harvard Medical School is on a crusade to bring personal genomics to the masses. "For $\$ 1,000$ you should be able to get $1 \%$ of your genome this year," says Church. Statements like this are fueling the fires of what is sure to be a new revolution in medicine and biology, the 


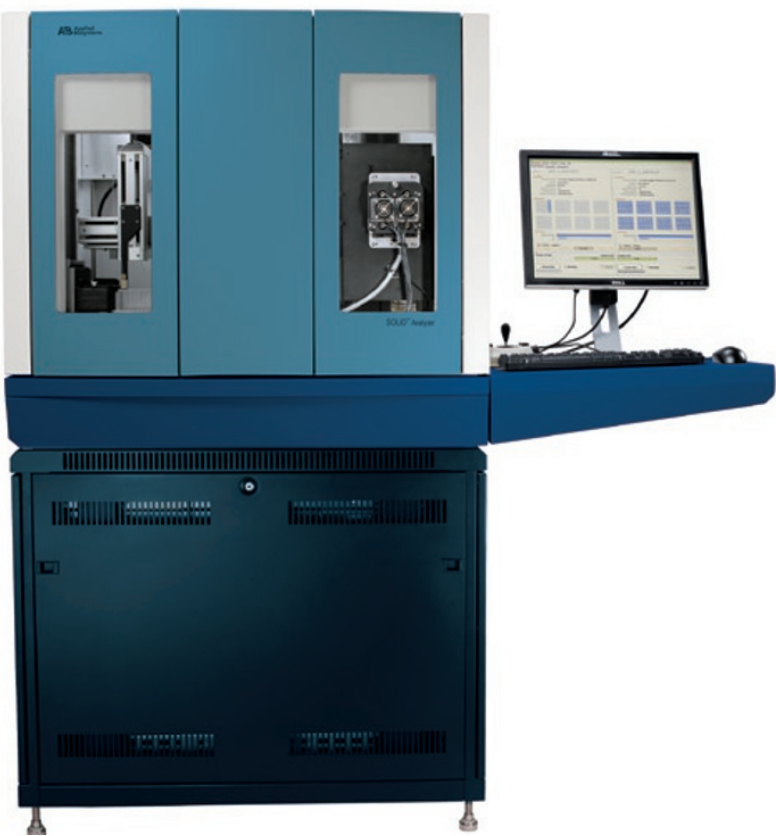

The SOLiD system from Applied Biosystems is an example of next-generation sequencing technology. (Image courtesy of Applied Biosystems.)

era of personal genomics (for additional discussion of technology behind personal genomics see the Technology Feature on personal genomics in the October 4, 2007 issue of Nature). Church notes that this $1 \%$ of the genome might yield $98 \%$ of the information regarding positions that cause changes in traits.

For personal genomics to succeed, a very large number of single molecules needs to be analyzed and examined. Church's lab has developed an emulsion-based method known as 'polony' sequencing to do just this. In the polony-which is just short for polymerase colony-method, DNA molecules are initially attached to either beads or glass in a 1:1 ratio, similar to BEAMing. If amplification is desired, a polymerase is used to amplify the attached DNA molecules, in a PCR-style reaction, providing more template and allowing for a greater signal-to-noise ratio.

The polony technology is now showing its versatility. "The polony concept includes most of the working methods right now for next-generation sequencing," says Church. And now polonies are also being applied to transcriptional analysis in single cells with very dramatic results.

Recently, Church and colleagues reported a new method called polony multiplex analysis of gene expression (PMAGE) ${ }^{4}$. PMAGE isolates short sequence tags from transcripts, in a process similar to serial analysis of gene expression (SAGE), using specialized endonucleases. These tags are then amplified by emulsion PCR and the polony beads are immobilized on a support for sequencing. The observed number of tags provides a direct measure of the transcript abundance. Using this method with cardiac tissues of normal mice and mice with hypertrophic cardiacmyopathy, the group analyzed almost 18,000 gene transcripts and identified several differentially regulated genes ${ }^{4}$.

PCR is now capable of single-cell and single-molecule exploration. The road to the single-molecule analysis has resulted in giant leaps in area of digital genetic analysis. The question of whether the scale will tip in favor of digital is only going to be answered over the next couple years. But one thing is for certain: whichever way it tips, scientists around the globe are going to be the beneficiaries.

1. Sanchez, J.A., Pierce, K.E., Rice, J.E. \& Wangh, L.J. Proc. Natl. Acad. Sci. USA 101, 1933-1938 (2004).

2. Raj, A., Peskin, C.S., Tranchina, D., Vargas, D.Y. \& Tyagi, S. PLOS Biol. 4, e309 (2006).

3. Diehl, F. et al. Proc. Natl. Acad. Sci. USA 102 , 16368-16373 (2005).

4. Kim, J.B. et al. Science 316, 1481-1484 (2007).

\section{Nathan Blow is Technology Editor for \\ Nature and Nature Methods \\ (n.blow@boston.nature.com).}




\section{SUPPLIERS GUIDE: COMPANIES OFFERING PCR REAGENTS AND SYSTEMS}

\section{Company}

Abgene

Agilent Technologies

Alpha Laboratories

Ambion, Inc.

Applied Biosystems

Beckman Coulter

Bioneer

Biopioneer Inc.

BioRad

BioTrove

Cepheid

Clontech

Corbett Life Sciences

EMD Biosciences

Epicentre Biotechnologies

Eppendorf

Exiqon

Finnzymes

Fluidigm

GE Healthcare

Genomas LLC

GenScript

Idaho Technologies

Integrated DNA Technologies

Invitrogen

MWB Biotech

New England Biolabs

Perkin Elmer Life Sciences

Premier Biosoft International

Promega

QBioGene

Qiagen

Quanta Biosciences

$R$ \& D Systems

Roche-Applied Science

Rubicon Genomics

Scigenix

Sigma Aldrich

Smiths Detection

Stratagene

Super Array

TakaRa Bio Inc.

Techne

Thermo Fisher Scientific

Toyobo

Transgenomics

USB

US Genomics

Wako Chemicals USA

\section{Web address}

http://www.abgene.com

http://www.agilent.com

http://www.alphalabs.co.uk

http://www.ambion.com

http://www.appliedbiosystems.com/

http://www.beckmancoulter.com

http://us.bioneer.com/

http://www.biopioneerinc.com

http://www.bio-rad.com

http://www.biotrove.com

http://www.cepheid.com

http://www.clontech.com

http://www.corbettlifescience.com

http://www.emdbiosciences.com

http://www.epibio.com

http://www.eppendorf.com

http://www.exiqon.com

http://www.finnzymes.com/

http://www.fluidigm.com

http://www.gehealthcare.com

http://www.genomas.net

http://www.genscript.com

http://www.idahotech.com

http://www.idtdna.com

http://www.invitrogen.com

http://www.mwg-biotech.com

http://www.neb.com

http://www.perkinelmer.com

http://www.premierbiosoft.com

http://www.promega.com

http://www.qbiogene.com

http://www1.qiagen.com

http://www.quantabio.com

http://www.rndsystems.com

http://www.roche.com

http://www.rubicongenomics.com

http://www.scigenix.com

http://www.sigmaaldrich.com

http://www.smithsdetection.com

http://www.stratagene.com

http://www.superarray.com

http://www.takara-bio.com

http://www.techne.com

http://www.thermofisher.com/

http://www.toyobo.co.jp

http://www.transgenomic.com

http://www.usbweb.com

http://www.usgenomics.com

http://www.wakousa.com 